\title{
Shenzhuo Formula Treatment in Patients With Macroalbuminuria Secondary to Diabetic Kidney Disease: Protocol Update and Statistical Analysis Plan
}

\section{Yu-Wei}

Beijing University of Chinese Medicine https://orcid.org/0000-0002-4460-9323

\section{Ying Zhang}

Beijing University of Chinese Medicine

Yanbo Li

Beijing University of Chinese Medicine

\section{Ze Yang}

China Academy of Traditional Chinese Medicine Guanganmen Hospital: China Academy of Chinese Medical Sciences Guanganmen Hospital

\section{Xinmiao Wang}

China Academy of Traditional Chinese Medicine Guanganmen Hospital: China Academy of Chinese Medical Sciences Guanganmen Hospital

Yi-Shan Huang ( $\square$ bucmyishan@163.com )

China Academy of Traditional Chinese Medicine Guanganmen Hospital: China Academy of Chinese Medical Sciences Guanganmen Hospital

\section{Lin-Hua Zhao}

China Academy of Traditional Chinese Medicine Guanganmen Hospital: China Academy of Chinese Medical Sciences Guanganmen Hospital

\section{Xiaolin Tong}

China Academy of Traditional Chinese Medicine Guanganmen Hospital: China Academy of Chinese Medical Sciences Guanganmen Hospital

\section{Research Article}

Keywords: Statistical analysis plan, Diabetic kidney disease, Shenzhuo Formula, Irbesartan, clinical effectiveness

Posted Date: August 27th, 2021

DOI: https://doi.org/10.21203/rs.3.rs-717285/v1

License: (9) (1) This work is licensed under a Creative Commons Attribution 4.0 International License. Read Full License 


\section{Abstract}

\section{Background}

Diabetic kidney disease (DKD), as one of the significant complications of diabetes, has been the focus of attention. Our previous retrospective study indicated that Shenzhou Formula (SZF) might reduce the macroalbuminuria secondary to the DKD.

\section{Methods and design}

This trial is a 24-week, randomized, multicentric, double-blinded, double-dummy clinical trial. 120 DKD patients (60 in each group) will be randomly divided randomized into two groups: SZF+ Irbesartan Simulator or Irbesartan + SZF Simulator. 24-h UP is the primary outcome measure is. The secondary outcome measures include Serum creatinine, estimated glomerular filtration rate, urinary albumin excretion rate, improvement in TCM symptoms, FBG, 2-h postprandial plasma glucose, HbA1C, Cholesterol, triglycerides, $\mathrm{HDL}$, LDL, blood pressure, albumin to creatinine ratio, and the audit of diabetes-dependent quality of life 19. Our recruitment began in May 2015, and we have enrolled 100 participants in this ongoing study, with a designed maximum sample size of 120 . The interim results have been reviewed at $\mathrm{N}=60$ and continuing recruitment was recommended. This statistical analysis plan includes our approach to impute missing data, analysis primary, secondary outcomes, and safety endpoints.

\section{Conclusion}

This SAP will standardize the clinical trial's statistical analysis, avoid outcome selective reporting bias and data-driven analysis. This trial will provide further clinical evidence for SZF treatment of macroalbuminuria secondary to DKD.

\section{Trial registration}

Chinese Clinical Trial Registry, ID: ChiCTR-ICR-15006311, registered on 26 May 2013. http://www.chictr.org.cn/showproj.aspx? proj $=10862$

\section{Background}

Diabetic kidney disease (DKD) is a leading cause of chronic kidney disease (CKD) in the developed countries and is rapidly becoming the leading cause in developing countries [1]. As one of the most common complications of diabetes mellitus, DKD has been reported in $20-40 \%$ of diabetic patients in China and has been the leading cause of end stage renal disease(ESRD) in the middle-aged and elderly people replacing chronic glomerulonephritis [2-4]. Despite the increasing use of renin-angiotensinaldosterone system(RAAS) inhibitors in recent years, the prevalence of diabetic nephropathy has not significantly changed $[5,6]$. Therefore, there is an urgent need to explore drugs that can effectively prevent DKD occurrence and development.

The treatment of DKD by traditional Chinese medicine (TCM) has accumulated extensive clinical experience in recent years. TCM and angiotensin receptor blockade (ARB) treatment combination can reduce proteinuria in type 2 diabetes [7]. The treatment reported in our previous retrospective study revealed that Shenzhou Formula (SZF) could improve glomerular filtration rate (GFR) and reduce 24-h urinary protein (24h-UP) [8]. Animal experiments also showed that TCMs have protective effects on diabetic renal lesions [9]. However, the impact of SZF versus RAAS blockers in DKD remains very limited and thus requires further exploration.

We designed and implemented the trial to investigate the efficacy of and safety of SZF and irbesartan in treating DKD patients with macroalbuminuria. We hypothesized that the treatment with SZF alone could alter the macroalbuminuria secondary to diabetic kidney disease. Shenzhou Formula treatment in patients with macroalbuminuria secondary to diabetic kidney disease is a 24week, randomized, multicentric, double-blinded, double-dummy, controlled clinical trial. The research protocol was previously reported [10]. The original protocol provides more details on the trial rationale, eligibility criteria, interventions, concomitant treatments, study visits, and data management.

Here, we describe the study's pre-determined statistical analysis plan (SAP), which will be applied when the trial ends, to prevent reporting bias and data-driven interpretation for results. The SAP (Version 2.0 20180626) was accomplished before the data analysis, and the investigators will adhere to the requirement in analyzing the data, according to "Guidelines for the Content of Statistical Analysis Plans in Clinical Trials" published in JAMA [11] (See Additional file 1 populated checklist). It includes our 
approach to imputing missing data, our approach to analyzing primary, secondary outcomes, and including safety, baseline analyzes. We will also report adverse effects in total.

\section{Study Methods}

\subsection{Trial design}

SZF treatment in patients with macroalbuminuria secondary to diabetic kidney disease is a clinical trial conducted by 14 centers, in which 120 subjects will be recruited. In the protocol, we specified that we would include 120 subjects in 9 hospitals. We added five centers to speed up the trial, including The Second Affiliated Hospital of Shaanxi University of TCM, Xianyang; Zhengzhou hospital of TCM, Zhengzhou; Hangzhou Hospital of TCM, Hangzhou; The Affiliated Hospital to Changchun University of Chinese Medicine, Changchun; Zhejiang Hospital of TCM, Hangzhou. The newly added centers have passed the ethical review by the Ethics Committee of Guang'anmen Hospital. All investigators received intensive training on conducting the research and intervention before the recruitment to ensure the quality. The flow diagram shown in Figure.1 provides a brief description of the research process. The protocol has defined the sample size calculation [10].

\subsection{Randomization and Blinding}

Participants included in the study will be randomized using blocked randomization stratified by centering a 1:1 ratio to receive SZF plus Irbesartan Simulator or Irbesartan plus SZF Simulator 24weeks. According to the center of each layer, select the appropriate length. With the PROC PLAN process statement of SAS 9.4 (SAS/STAT Software 9.4, SAS Institute, Inc., Cary, NC), the random arrangement (random coding table) of 120 subjects was generated given the number of seeds. The Institute of Basic Research in Clinical Medicine of the China Academy of Chinese Medical Sciences provided the random arrangement. So did the 24-hour emergency code break and medical information. Participants, investigators, and statisticians will be unaware of treatment assignments.

SZF drugs and SZF mimic are precisely the same shape and smell. The taste, smell, color are also entirely consistent when dissolved in water. Irbesartan capsules and mimics have a consistent condition; the scent and appearance remain consistent after opening the capsule.

\subsection{Interim analysis}

Interim analyzes occurred when 60 patients completed the 24-week treatment, which is $50 \%$ of the planned number of cases, by independent biostatisticians. The primary, secondary outcomes and safety assessments will be tested to compare the SZF and irbesartan effect and safety. If there were concerns about the effect and safety of participants, Data Monitoring Committee would recommend continuing, stopping, or modifying the trial.

A recommendation of trial termination would be considered by the data management, clinical trial institutions, ethics committees, and sponsors if the corresponding difference in treatment effects were unlikely to be reached or intolerable adverse effects occurred. The detailed result will remain confidential to the investigators. The interim analysis occurred in September 2018, and continuing recruitment was recommended.

\subsection{Criteria for trial discontinuation}

Trial abortion refers to discontinuing a full trial halfway when the clinical trial has not ended as organized. The purpose of trial discontinuation is to protect subject equity, guarantee the trial quality, and avoid unnecessary economic losses. If any situation described below occurred, the trial would be terminated.

(1) Serious safety issues occur in the trial, and the trial should be promptly discontinued.

(2) Drug treatments found to be too inadequate or even ineffective in the trial to be of clinical value should be suspended, avoiding patient treatment delays and economic losses. 
(3) The clinical trial protocol is found to have a significant lapse or a well-designed protocol that has undergone substantial deviations in implementation, which makes it challenging to evaluate the drug effect.

(4) The sponsor requests discontinuation (e.g., reasons for funding, administration, etc.).

(5) Withdrawal of the trial by the administration, etc.

\section{Statistical principles}

\subsection{0verall principles}

All the statistical analysis tests will be implemented based on two-side tests with a nominal alpha level of 0.05 by the SAS [12] after the completion of the trial. All confidence intervals (Cls) reports will be $95 \%$ Cls. For each outcome, the null hypothesis is that there is no difference between the intervention groups.

The description of measurement indicators will calculate the mean, standard deviation, median, minimum, maximum, interquartile range (Q3-Q1).

Continuous variables satisfying normality will be described by the mean and standard deviation, and the median and interquartile range when distribution did not follow normality. The counting indicators will be presented as the number and percentage of each category.

Inter-group comparisons for metrics will be performed with the independent two-sample t-tests (normality, homogeneity of variance) or Wilcoxon rank-sum test (non-normality, heterogeneity of variance). Comparisons within a group will be carried out using the paired t-test (normality) or paired rank-sum test (non-normality). $\chi 2$ test or Fisher's exact probability will be needed for count metrics, and the Wilcoxon rank-sum test will be utilized for categorical variables. A generalized linear mixed model analysis (GLM) will be performed on the repeated measured outcome.

Suppose there is an imbalance in the baseline of important indicators on the efficacy analysis, such as age, gender, and the concomitant treatment. In that case, an adjustment will be required before the efficacy comparison. The covariance method will be used in continuous variables, and logistic regression analysis will be used in count metrics and hierarchical metrics.

\subsection{Definition of analysis sets}

\subsubsection{Full Analysis Set, FAS}

The patients who have accepted treatment at least once and have the primary objective after treatment will be included in the FAS.

\subsubsection{Per Protocol Set, PPS}

The subjects will be included in the per protocol set, selected in the FAS and received at least $50 \%$ treatment scheduled with no major protocol violations. The PPS will be determined previous to the unblinding of the data for the final analysis and will include:

All visits planned and the main data completed.

The inclusion satisfied and the exclusion inapplicable.

No significant violations of the trial protocol occurred (described in Adherence and Protocol deviations).

\subsubsection{Safety Set, SS}

The safety analysis will analyze all the patients who used the intervention drug at least once and had one safety assessment.

\subsection{Adherence and Protocol deviations}

Adherence is determined by the participants each visit point attending and the medication adherence. We will describe the proportion of participants by using frequency distributions for both groups. The medication compliance will be calculated in two 
groups separately and compared between groups according to the ratio of medicine actual taken, and the drug should be taken. The medicine distributed to the patients and the medicine left untaken will be recorded every four weeks.

Significant protocol violations, including those that could affect the primary measures, like taking protocol prohibited medicine, those which present a safety risk to the participants, and/or those that are of ethical concern.

\section{Trial population}

\subsection{Eligibility criteria}

Inclusion and exclusion criteria used in the trial are presented in Table 2 of the protocol. The participants with DKD need to have macroalbuminuria and have well-controlled Serum creatinine, blood pressure, and HbA1c. Participants are excluded, including those who take the potassium-sparing diuretics or with Type 1 diabetes.

\subsection{Case enrollment and completion}

Enrollment and the number of completed cases will be summarized for each center. The numbers of enrolled cases and each analyzed data set will be described. Participants may withdraw from the trial intervention and/or fail to provide the follow-up data. In addition, participants may withdraw the trial consent. We will report these items (Table 1), and the total dropout rate will be compared between the two groups.

\subsection{Baseline characteristics}

The baseline characteristics of all participants in both groups will be outlined in Table 2. The table will describe the following variables: age, height, weight, BMI, female sex, nationality, heart rate, systolic blood pressure, diastolic blood pressure, mean arterial pressure, respiration rate, drug or constitution allergic history, comorbid conditions, concomitant treatment. The sample will be described according to the full analysis set. The data of the two groups of subjects will be compared between groups to determine the equality of the baseline values of the two groups.

\section{Analysis methods}

\subsection{Outcome measures analyzes}

\subsubsection{Primary outcome analyses}

The primary analysis will be conducted in the full analysis set and per protocol set. The effect of SZF and its application in reducing urine protein, which is evaluated by the $24-\mathrm{h} \mathrm{UP}(\mathrm{mg} / 24 \mathrm{~h})$, is the primary concern. The distribution and changes of $24-\mathrm{h}$ UP at week $0,4,8,12,16,20$, and week 24 of medication will be described. Intra-group and inter-group comparisons will be made. The distributions of qualitative judgments of 24-h UP at weeks $4,8,12,16,20$, and 24 will be described and compared between groups. Table 3 is provided for reference in qualitatively judging the variation. The efficiency rate will be calculated by summing the rate of clinical control, obvious therapeutic effect, and with therapeutic effect (Table 4).

\subsubsection{Secondary outcome analyses}

The secondary outcome including Serum creatinine (SCr, umol/L) and estimated Glomerular filtration rate (eGFR, $\mathrm{mL} /$ (min•1.73m2), using CKD-EPI creatinine equation[13] ), urinary albumin excretion rate (UAER, $\mu \mathrm{g} / \mathrm{min})$,improvement in TCM symptoms, fasting blood glucose(FBG) (mmol/L) and 2-h postprandial plasma glucose(2-h PG, mmol/L), $\mathrm{HbA1C}(\%)$, Cholesterol(mmol/L), triglycerides(mmol/L), $\mathrm{HDL}(\mathrm{mmol} / \mathrm{L}), \mathrm{LDL}(\mathrm{mmol} / \mathrm{L})$, blood pressure $(\mathrm{mmHg})$, albumin to creatinine ratio(ACR)(mg/g) and the audit of diabetes-dependent quality of life 19 (ADDQoL)[14] and it will be conducted in the FAS and PPS. The distribution and changes of all the secondary outcomes at the baseline and the visit point will be compared between groups and within the group (Table 5).

Describe and compare the TCM symptom (physical and mental fatigue, dry mouth and throat, weakness and soreness of the lower back and knees, qi deficiency and listlessness, spontaneous perspiration, night sweat, dysphoria in chest, palms, and soles, dark purplish lips, edema) scores and the total score from the baseline and 24 weeks after medication in both groups. 
The improvement of TCM syndromes will be judged according to the symptom score method, which is conducted according to the Nimodipine method [15]. Efficacy index $=$ (Score before treatment - Score after treatment) $/$ Score before treatment $\times 100 \%$. The criteria are as follows:

Clinical contro/ is defined as almost all symptoms disappeared after the treatment (the efficacy index $\geq 95 \%$ ). The obvious therapeutic effect means that most clinical signs show marked improvement (the efficacy index $\geq 70 \%$ ). With therapeutic effect means the relief of the symptoms has been experienced (the efficacy index $\geq 30 \%$ ). Invalid: no significant improvement or symptom exacerbation (the efficacy index $₫ 30 \%$ ). The clinical efficiency will be calculated with the formula: Clinical efficiency = (Clinical control case number + Obvious therapeutic effect case number + With therapeutic effect number) / total case number $\times$ $100 \%$.

The clinical efficiency of TCM syndrome scores after 24 weeks of treatment will be calculated for both groups (Table 6).

\subsection{Safety analyzes}

The safety analysis will analyze all the patients in Safety Set. The laboratory index would be listed one by one if the patients had their laboratory findings conversed from normal to abnormal or aggravated and the abnormal liver and renal function occurred during the treatment, as well as the judgments of the association between the conversion and medications. The type, severity, occurrence, and relationship to intervention drug of all adverse events during treatment will be tabulated (Table 7). The number and frequency of adverse events will be calculated for both groups. The occurrence of serious adverse events and the discontinuation followed will be recorded in detail. The incidence of adverse effects(AEs) will be compared between groups.

Any AE will be classified into four levels: mild (Mild discomfort, which the participants could tolerate with no special treatment.), moderate (Moderate pain, which the participants could not handle and need special treatment with a direct impact on recovery.), and severe AEs (life-threatening or lead to death, hospitalization, extended hospitalization, to lasting or severe disability, incapacity or congenital anomaly.)

The safety assessment includes the routine blood, urine, and stool tests, electrocardiogram, liver function, renal function inspected at baseline, week 12, and week 24 (Table 8). Safety analysis will describe the distribution by qualitative judgment, which is counting the number of cases and their proportion of abnormal indexes by cross-tabulation.

\subsection{Imputation of missing data}

Multiple imputations will be used for subjects with missing data on the primary outcome. For the secondary and safety outcomes that may miss, the results will be analyzed according to the actual data instead of imputing the missing values or adjusting the model for missing data. Furthermore, we will conduct a per protocol analysis which excluded those with missing outcome data.

\section{Changes to the original SAP}

The original SAP was created based on the initial study protocol and related planned analyses. The focal point of the study protocol update and/or SAP reversion since the first version, including the additional ACR measurement, the ADDQoL were added as secondary outcomes, and an interim analysis was implemented to evaluate measures of effectiveness and safety.

\section{Conclusions}

The present study is a 24-week, randomized, multicentric, double-blinded, double-dummy clinical trial. Unbiased evaluation will be conducted on the clinical data in the trial to increase the credibility of the results and bring substantial evidence for further studies in the treatment of DKD.

\section{Status}

The trial commenced in August 2014 and is scheduled to be completed in June 2022. Currently, 100 patients have been recruited.

\section{References to files}


Data Management Plan and Statistical Master File developed and kept by a third-party statistical company. Trial Master Files are developed and retained by the Guang'anmen Hospital (principal investigators unit). The SOPs were developed collectively by the principal investigators and the participating centers and were preserved separately.

\section{Abbreviations}

\begin{tabular}{|ll|}
\hline DKD & Diabetic kidney disease \\
\hline SZF & Shenzhou Formula \\
\hline CKD & chronic kidney disease \\
\hline ESRD & end stage renal disease \\
\hline RASS & Renin-Angiotensin-Aldosterone System \\
\hline TCM & traditional Chinese medicine \\
\hline ARB & angiotensin receptor blockade \\
\hline GFR & glomerular filtration rate \\
\hline 24h-UP & 24-h urinary protein \\
\hline SAP & statistical analysis plan \\
\hline Cls & confidence intervals \\
\hline GLM & generalized linear mixed model analysis \\
\hline FAS & Full Analysis Set \\
\hline PPS & Per Protocol Set \\
\hline SS & Safety Set \\
\hline UAER & urinary albumin excretion rate \\
\hline FBG & fasting blood glucose \\
\hline 2-h PG & 2-h postprandial plasma glucose \\
\hline ADDQoL & the audit of diabetes-dependent quality of life 19 \\
\hline AEs & adverse effects \\
\hline ACR & albumin to creatinine ratio \\
\hline
\end{tabular}

\section{Declarations}

\section{Ethics approval and consent to participate}

The trial was approved by the Ethics Committee of Guang'anmen Hospital prior to the recruitment of patients.

\section{Consent for publication}

The manuscript doesn't contain any individual person's data in any form.

\section{Availability of data and materials}

Not applicable. The trials discussed in this publication will be published or have been published separately. This project did not generate data or materials.

Availability of data and materials 
Data sharing is not applicable to this article as no datasets were generated or analyzed in this manuscript.

\section{Competing interests}

The authors declare that they have no no conflicts of interests

\section{Funding}

This study is supported by the Fundamental Research Funds for the Central Public Welfare Research Institutes (No. ZZ0808004), and Guang's Anmen Hospital of the China Academy of Chinese Medical Science (No. 2015 s325).

\section{Authors' contributions}

All authors critically revised and contributed to the content of the updated SAP and this manuscript and approved the final submitted version.

\section{Acknowledgments}

The authors are grateful to the Data Monitoring Company (Beijing Created Medicine Technology Co.『LTD) for current and initial statistical support. We also deliver our thanks to all the staff for their valuable supports in participant recruitment.

\section{References}

1. KDOQI Clinical Practice Guidelines and Clinical Practice Recommendations for Diabetes and Chronic Kidney Disease. American journal of kidney diseases: the official journal of the National Kidney Foundation.2007;49(2 Suppl 2): S12-154.

2. The Microvascular Complications Group of Chinese Diabetes Association. Chinese clinical practice guideline of diabetic kidney disease. Chinese Journal of Diabetes Mellitus. 2019; 11(01): 15-28.

3. Hou F, Jiang J, Chen J, Yu X, Zhou Q, Chen P, et al. China collaborative study on dialysis: a multi-centers cohort study on cardiovascular diseases in patients on maintenance dialysis. BMC nephrology.2012;13(94).

4. Chinese guidelines for diagnosis and treatment of Diabetic Kidney Disease . Chinese Journal of Nephrology.2021;37(03):255304

5. De B, Rue T, Hall Y, Heagerty P, Weiss N, Himmelfarb J.Temporal trends in the prevalence of diabetic kidney disease in the United States. JAMA.2011;305(24):2532-2539.

6. Afkarian M, Zelnick L, Hall Y, Heagerty P, Tuttle K, Weiss N, et al. Clinical Manifestations of Kidney Disease Among US Adults With Diabetes, 1988-2014. JAMA.2016;316(6):602-610

7. Xiang L, Jiang P, Zhou L, Sun X, Bi J, Cui L, et al.Additive Effect of Qidan Dihuang Grain, a Traditional Chinese Medicine, and Angiotensin Receptor Blockers on Albuminuria Levels in Patients with Diabetic Nephropathy: A Randomized, ParallelControlled Trial. Evidence-based complementary and alternative medicine: eCAM.2016;2016:1-8.

8. Tian J, Zhao L, Zhou Q, Liu W, Chen X, Lian F, et al. Efficacy of Shenzhuo formula on diabetic kidney disease: a retrospective study. Journal of traditional Chinese medicine.2015;35(5):528-536.

9. Zhou J, Pan J, Xiang, Wang Q, Tong Q, Fang J, et al. Xiaokeyinshui extract combination, a berberine-containing agent, exerts anti-diabetic and renal protective effects on rats in multi-target mechanisms. Journal of Ethnopharmacology.2020;262:1-11.

10. Zhao X, Zhang Y, He X, Chen H, Wang Z, Guo J, et al.Chinese herbal medicine Shenzhuo Formula treatment in patients with macroalbuminuria secondary to diabetic kidney disease: study protocol for a randomized controlled trial.

Trials.2018;19(1):200.

11. Gamble C, Krishan A, Stocken D, Lewis S, Juszczak E, Doré C, et al. Guidelines for the Content of Statistical Analysis Plans in Clinical Trials. JAMA.2017;318(23):2337-2343.

12. Vidrine D, Frank-Pearce S, Vidrine J, Tahay P, Marani S, Chen S, et al.Efficacy of Mobile Phone-Delivered Smoking Cessation Interventions for Socioeconomically Disadvantaged Individuals: A Randomized Clinical Trial. JAMA Intern

Med.2019;179(2):167-174. 
13. Levey A, Stevens L, Schmid C, Zhang Y, Castro A, Feldman H, et al. A new equation to estimate glomerular filtration rate. Annals of internal medicine.2009;150(9):604-612.

14. Fung C, Wan E, Yu C, Wong C. Validity and reliability of the 19-item Audit of Diabetes-Dependent Quality of Life (ADDQoL-19) questionnaire in Chinese patients with type 2 diabetes mellitus in primary care. Quality of life research: an international journal of quality of life aspects of treatment, care and rehabilitation.2016;25(9):2373-2378

15. Zhao L, Wang J, Li H, Che J, Ma N, Cao B. Safety and Efficacy of Tianfoshen Oral Liquid in Non-Small Cell Lung Cancer Patients as an Adjuvant Therapy. Evidence-based complementary and alternative medicine: eCAM.2019;2019:1-7.

\section{Tables}

Table 1. Withdrawals

\begin{tabular}{|llll|}
\hline Num & Withdrew at... & Reason withdrew & Type of withdrawal \\
\hline 1 & $\mathrm{xx}$ & $\mathrm{xx}$ & e.g. withdrew consent \\
\hline 2 & $\mathrm{xx}$ & $\mathrm{xx}$ & $\begin{array}{l}\text { e.g. stopped intervention } \\
\text { early, provided data at follow-up }\end{array}$ \\
\hline$\ldots$ & $\mathrm{xx}$ & $\mathrm{xx}$ & e.g. stopped intervention early, lost to follow-up \\
\hline$\ldots$ & $\mathrm{xx}$ & $\mathrm{xx}$ & e.g. completed intervention, lost to follow-up \\
\hline $\mathrm{n}$ & $\mathrm{xx}$ & $\mathrm{xx}$ & $\mathrm{xx}$ \\
\hline
\end{tabular}

Table 2. Baseline characteristics of participants prior to treatment 


\begin{tabular}{|c|c|c|c|c|}
\hline Characteristic & $\begin{array}{l}\text { SZF, number, central } \\
\text { tendency (variability) }\end{array}$ & $\begin{array}{l}\text { Irbesartan, number, central } \\
\text { tendency (variability) }\end{array}$ & $\begin{array}{l}\text { All participants, number, } \\
\text { central tendency (variability) }\end{array}$ & $p$ Value \\
\hline & $n=\mathrm{xx}$ & $n=\mathrm{xx}$ & $n=\mathrm{xx}$ & \\
\hline \multicolumn{5}{|l|}{ Demographics } \\
\hline Age(years) ${ }^{a}$ & $x x(x x)$ & $x x(x x)$ & $x x(x x)$ & $x x$ \\
\hline Height $(m)^{a}$ & $x x(x x)$ & $x x(x x)$ & $x x(x x)$ &.$x x$ \\
\hline Weight $(\mathrm{kg})^{\mathrm{a}}$ & $x x(x x)$ & $x x(x x)$ & $x x(x x)$ & $x x$ \\
\hline $\mathrm{BMI}(\mathrm{kg} / \mathrm{m} 2)^{\mathrm{a}}$ & $x x(x x)$ & $x x(x x)$ & $x x(x x)$ &.$x x$ \\
\hline Female sex $(n, \%)^{b}$ & $x x(x x \%)$ & $x x(x x \%)$ & $x x(x x \%)$ &.$x x$ \\
\hline Nationality(China, other) ${ }^{b}$ & $x x(x x \%)$ & $x x(x x \%)$ & $x x(x x \%)$ &.$x x$ \\
\hline \multicolumn{5}{|l|}{ Vital signs } \\
\hline $\begin{array}{l}\text { Heart rate(beats per } \\
\text { minute) })^{\mathrm{a}}\end{array}$ & $x x(x x)$ & $x x(x x)$ & $x x(x x)$ & $x x$ \\
\hline $\begin{array}{l}\text { Systolic blood pressure } \\
(\mathrm{mmHg})^{\mathrm{a}}\end{array}$ & $x x(x x)$ & $x x(x x)$ & $x x(x x)$ &.$x x$ \\
\hline $\begin{array}{l}\text { Diastolic blood pressure } \\
(\mathrm{mmHg})^{\mathrm{a}}\end{array}$ & $x x(x x)$ & $x x(x x)$ & $x x(x x)$ &.$x x$ \\
\hline $\begin{array}{l}\text { Mean arterial pressure } \\
(\mathrm{mmHg})^{\mathrm{a}}\end{array}$ & $x x(x x)$ & $x x(x x)$ & $x x(x x)$ &.$x x$ \\
\hline $\begin{array}{l}\text { Respiration } \\
\text { rate(breath/min) }\end{array}$ & $x x(x x)$ & $x x(x x)$ & $x x(x x)$ &.$x x$ \\
\hline $\begin{array}{l}\text { Drug or constitution } \\
\text { allergic history (yes or } \\
\text { no) }\end{array}$ & $x x(x x \%)$ & $x x(x x \%)$ & $x x(x x \%)$ &.$x x$ \\
\hline \multicolumn{5}{|l|}{ Comorbid conditions(n\%) } \\
\hline Diabetic retinopathy ${ }^{\mathrm{b}}$ & $x x(x x \%)$ & $x x(x x \%)$ & $x x(x x \%)$ &.$x x$ \\
\hline \multicolumn{5}{|l|}{$\ldots$} \\
\hline \multicolumn{5}{|l|}{ Concomitant treatment } \\
\hline$\ldots$ & $x x(x x \%)$ & $x x(x x \%)$ & $x x(x x \%)$ &.$x x$ \\
\hline
\end{tabular}

a Number, mean, standard deviation

b Number, percentage.

Table 3. 24-h UP qualitative judgments classification standard

\begin{tabular}{|ll|}
\hline Qualitative Judgment & Standard \\
\hline Clinical control & 24-h UP $\llbracket 0.5 \mathrm{~g}$ \\
\hline Obvious therapeutic effect & 24-h UP reduction $\geq 50 \%$ \\
\hline With therapeutic effect & 24-h UP reduction $\geq 20 \%$ \\
\hline Invalid & Fail to reach the standards above \\
\hline
\end{tabular}


Table 4. Analysis of primary outcome

\begin{tabular}{|lllll|}
\hline Time point & SZF, number, mean (SD) & Irbesartan, number, mean (SD) & Mean difference (95\% Cl) & $p$ Value \\
\hline visit at & $n=x x$ & $n=x x$ & $x x(x x$ to $x x)$ &.$x x$ \\
\hline Week 0 & $x x(x x)$ & $x x(x x)$ & $x x(x x$ to $x x)$ &.$x x$ \\
\hline Week 4 ${ }^{a}$ & $x x(x x)$ & $x x(x x)$ & $x x(x x$ to $x x)$ &.$x x$ \\
\hline Week 8 & $x x(x x)$ & $x x(x x)$ & $x x(x x$ to $x x)$ &.$x x$ \\
\hline Week 12 & $x x(x x)$ & $x x(x x)$ & $x x(x x$ to $x x)$ &.$x x$ \\
\hline Week 16 & $x x(x x)$ & $x x(x x)$ & $x x(x x$ to $x x)$ &.$x x$ \\
\hline Week 20 & $x x(x x)$ & &.$x x$ \\
\hline Week 24 & $x x(x x)$ & $x x(x x)$ & \\
\hline Overall SZF effect ${ }^{b}$ & & & & $. x x)$ \\
\hline
\end{tabular}

$a, b$ : $p$ values comparing between group differences at visit points post-randomization (a) and over the entire24-week trial (b: primary outcome).

Table 5. Secondary outcome analysis 


\begin{tabular}{|c|c|c|c|c|}
\hline Time point & SZF, number, mean (SD) & Irbesartan, number, mean (SD) & Mean difference (95\% Cl) & $p$ Value \\
\hline Scr at & $n=\mathrm{xx}$ & $n=\mathrm{xx}$ & & \\
\hline Week 0 & $x x(x x)$ & $x x(x x)$ & $x x(x x$ to $x x)$ &.$x x$ \\
\hline Week 4 & $x x(x x)$ & $x x(x x)$ & $x x(x x$ to $x x)$ &.$x x$ \\
\hline Week 8 & $x x(x x)$ & $x x(x x)$ & $x x(x x$ to $x x)$ &.$x x$ \\
\hline Week 12 & $x x(x x)$ & $x x(x x)$ & $x x(x x$ to $x x)$ &.$x x$ \\
\hline Week 16 & $x x(x x)$ & $x x(x x)$ & $x x(x x$ to $x x)$ &.$x x$ \\
\hline Week 20 & $x x(x x)$ & $x x(x x)$ & $x x(x x$ to $x x)$ &.$x x$ \\
\hline Week 24 & $x x(x x)$ & $x x(x x)$ & $x x(x x$ to $x x)$ &.$x x$ \\
\hline \multicolumn{2}{|c|}{ Overall SZF effect ${ }^{a}$} & & &.$x x$ \\
\hline eGFR at & $n=\mathrm{xx}$ & $n=\mathrm{xx}$ & & \\
\hline Week 0 & $x x(x x)$ & $x x(x x)$ & $x x(x x$ to $x x)$ &.$x x$ \\
\hline Week 4 & $x x(x x)$ & $x x(x x)$ & $x x(x x$ to $x x)$ &.$x x$ \\
\hline Week 8 & $x x(x x)$ & $x x(x x)$ & $x x(x x$ to $x x)$ &.$x x$ \\
\hline Week 12 & $x x(x x)$ & $x x(x x)$ & $x x(x x$ to $x x)$ &.$x x$ \\
\hline Week 16 & $x x(x x)$ & $x x(x x)$ & $x x(x x$ to $x x)$ &.$x x$ \\
\hline Week 20 & $x x(x x)$ & $x x(x x)$ & $x x(x x$ to $x x)$ &.$x x$ \\
\hline Week 24 & $x x(x x)$ & $x x(x x)$ & $x x(x x$ to $x x)$ &.$x x$ \\
\hline \multicolumn{2}{|c|}{ Overall SZF effect } & & &.$x x$ \\
\hline UAER at & $n=x x$ & $n=x x$ & & \\
\hline Week 0 & $x x(x x)$ & $x x(x x)$ & $x x(x x$ to $x x)$ &.$x x$ \\
\hline Week 4 & $x x(x x)$ & $x x(x x)$ & $x x(x x$ to $x x)$ &.$x x$ \\
\hline Week 8 & $x x(x x)$ & $x x(x x)$ & $x x(x x$ to $x x)$ &.$x x$ \\
\hline Week 12 & $x x(x x)$ & $x x(x x)$ & $x x(x x$ to $x x)$ &.$x x$ \\
\hline Week 16 & $x x(x x)$ & $x x(x x)$ & $x x(x x$ to $x x)$ &.$x x$ \\
\hline Week 20 & $x x(x x)$ & $x x(x x)$ & $x x(x x$ to $x x)$ &.$x x$ \\
\hline Week 24 & $x x(x x)$ & $x x(x x)$ & $x x(x x$ to $x x)$ &.$x x$ \\
\hline \multicolumn{2}{|c|}{ Overall SZF effect } & & &.$x x$ \\
\hline FBG & $n=x x$ & $n=x x$ & & \\
\hline Week 0 & $x x(x x)$ & $x x(x x)$ & $x x(x x$ to $x x)$ &.$x x$ \\
\hline Week 4 & $x x(x x)$ & $x x(x x)$ & $x x(x x$ to $x x)$ &.$x x$ \\
\hline Week 8 & $x x(x x)$ & $x x(x x)$ & $x x(x x$ to $x x)$ &.$x x$ \\
\hline Week 12 & $x x(x x)$ & $x x(x x)$ & $x x(x x$ to $x x)$ &.$x x$ \\
\hline Week 16 & $x x(x x)$ & $x x(x x)$ & $x x(x x$ to $x x)$ &.$x x$ \\
\hline Week 20 & $x x(x x)$ & $x x(x x)$ & $x x(x x$ to $x x)$ &.$x x$ \\
\hline Week 24 & $x x(x x)$ & $x x(x x)$ & $x x(x x$ to $x x)$ &.$x x$ \\
\hline
\end{tabular}




\begin{tabular}{|c|c|c|c|c|}
\hline Overall SZF effect & & & &.$x x$ \\
\hline $2 \mathrm{hPG}$ at & $n=\mathrm{xx}$ & $n=\mathrm{xx}$ & & \\
\hline Week 0 & $x x(x x)$ & $x x(x x)$ & $x x(x x$ to $x x)$ &.$x x$ \\
\hline Week 4 & $x x(x x)$ & $x x(x x)$ & $x x(x x$ to $x x)$ &.$x x$ \\
\hline Week 8 & $x x(x x)$ & $x x(x x)$ & $x x(x x$ to $x x)$ &.$x x$ \\
\hline Week 12 & $x x(x x)$ & $x x(x x)$ & $x x(x x$ to $x x)$ &.$x x$ \\
\hline Week 16 & $x x(x x)$ & $x x(x x)$ & $x x(x x$ to $x x)$ &.$x x$ \\
\hline Week 20 & $x x(x x)$ & $x x(x x)$ & $x x(x x$ to $x x)$ &.$x x$ \\
\hline Week 24 & $x x(x x)$ & $x x(x x)$ & $x x(x x$ to $x x)$ &.$x x$ \\
\hline Overall SZF effect & & & &.$x x$ \\
\hline $\mathrm{TC}$ at & $n=\mathrm{xx}$ & $n=\mathrm{xx}$ & & \\
\hline Week 0 & $x x(x x)$ & $x x(x x)$ & $x x(x x$ to $x x)$ &.$x x$ \\
\hline Week 12 & $x x(x x)$ & $x x(x x)$ & $x x(x x$ to $x x)$ &.$x x$ \\
\hline Week 24 & $x x(x x)$ & $x x(x x)$ & $x x(x x$ to $x x)$ &.$x x$ \\
\hline Overall SZF effect & & & &.$x x$ \\
\hline TG at & $n=\mathrm{xx}$ & $n=x x$ & & \\
\hline Week 0 & $x x(x x)$ & $x x(x x)$ & $x x(x x$ to $x x)$ &.$x x$ \\
\hline Week 12 & $x x(x x)$ & $x x(x x)$ & $x x(x x$ to $x x)$ &.$x x$ \\
\hline Week 24 & $x x(x x)$ & $x x(x x)$ & $x x(x x$ to $x x)$ &.$x x$ \\
\hline Overall SZF effect & & & &.$x x$ \\
\hline LDL at & $n=\mathrm{xx}$ & $n=\mathrm{xx}$ & & \\
\hline Week 0 & $x x(x x)$ & $x x(x x)$ & $x x(x x$ to $x x)$ &.$x x$ \\
\hline Week 12 & $x x(x x)$ & $x x(x x)$ & $x x(x x$ to $x x)$ &.$x x$ \\
\hline Week 24 & $x x(x x)$ & $x x(x x)$ & $x x(x x$ to $x x)$ &.$x x$ \\
\hline Overall SZF effect & & & &.$x x$ \\
\hline $\mathrm{HDL}$ at & $n=\mathrm{xx}$ & $n=\mathrm{xx}$ & & \\
\hline Week 0 & $x x(x x)$ & $x x(x x)$ & $x x(x x$ to $x x)$ &.$x x$ \\
\hline Week 12 & $x x(x x)$ & $x x(x x)$ & $x x(x x$ to $x x)$ &.$x x$ \\
\hline Week 24 & $x x(x x)$ & $x x(x x)$ & $x x(x x$ to $x x)$ &.$x x$ \\
\hline Overall SZF effect & & & &.$x x$ \\
\hline $\mathrm{HbA} 1 \mathrm{c}$ at & $n=\mathrm{xx}$ & $n=\mathrm{xx}$ & & \\
\hline Week 0 & $x x(x x)$ & $x x(x x)$ & $x x(x x$ to $x x)$ &.$x x$ \\
\hline Week 12 & $x x(x x)$ & $x x(x x)$ & $x x(x x$ to $x x)$ &.$x x$ \\
\hline Week 24 & $x x(x x)$ & $x x(x x)$ & $x x(x x$ to $x x)$ &.$x x$ \\
\hline Overall SZF effect & & & &.$x x$ \\
\hline BP at & $n=\mathrm{xx}$ & $n=\mathrm{xx}$ & & \\
\hline
\end{tabular}




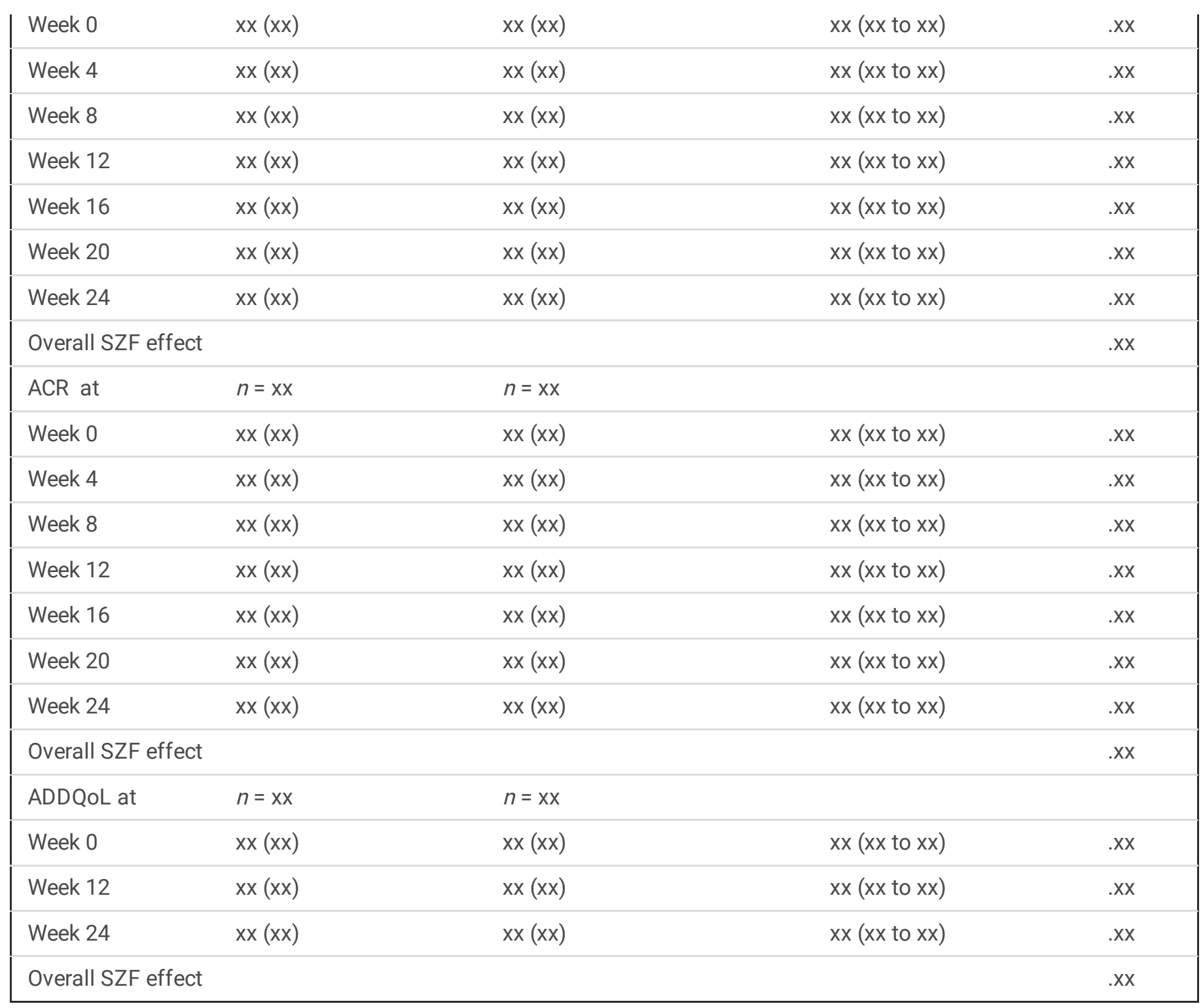

${ }^{a} p$ values comparing between-group differences over the entire 24-week trial

Table 6. TCM syndrome scores analysis 


\begin{tabular}{|c|c|c|c|c|c|c|c|}
\hline & & SZF & SZF & & Irbesartan & Irbesartan & \\
\hline & & $\begin{array}{l}\text { Week } \\
0\end{array}$ & $\begin{array}{l}\text { Week } \\
24\end{array}$ & $p$ Value & Week 0 & Week 24 & $p$ Value \\
\hline & & $\begin{array}{l}n= \\
\mathrm{xx}\end{array}$ & $n=\mathrm{xx}$ & & $n=\mathrm{xx}$ & $n=\mathrm{xx}$ & \\
\hline \multirow[t]{9}{*}{$\begin{array}{l}\text { Evaluation of TCM } \\
\text { syndrome }\end{array}$} & Physical and mental fatigue & $\begin{array}{l}x x \\
(x x)\end{array}$ & $\begin{array}{l}x x \\
(x x)\end{array}$ &.$x x$ & $x x(x x)$ & $x x(x x)$ &.$x x$ \\
\hline & Dry mouth and throat & $\begin{array}{l}x x \\
(x x)\end{array}$ & $\begin{array}{l}x x \\
(x x)\end{array}$ &.$x x$ & $x x(x x)$ & $x x(x x)$ &.$x x$ \\
\hline & $\begin{array}{l}\text { Weakness and soreness of the } \\
\text { lower back and knees }\end{array}$ & $\begin{array}{l}x x \\
(x x)\end{array}$ & $\begin{array}{l}x x \\
(x x)\end{array}$ &.$x x$ & $x x(x x)$ & $x x(x x)$ &.$x x$ \\
\hline & Qi deficiency and listlessness & $\begin{array}{l}x x \\
(x x)\end{array}$ & $\begin{array}{l}x x \\
(x x)\end{array}$ &.$x x$ & $x x(x x)$ & $x x(x x)$ &.$x x$ \\
\hline & Spontaneous perspiration & $\begin{array}{l}x x \\
(x x)\end{array}$ & $\begin{array}{l}x x \\
(x x)\end{array}$ &.$x x$ & $x x(x x)$ & $x x(x x)$ &.$x x$ \\
\hline & Night sweat & $\begin{array}{l}x x \\
(x x)\end{array}$ & $\begin{array}{l}x x \\
(x x)\end{array}$ &.$x x$ & $x x(x x)$ & $x x(x x)$ &.$x x$ \\
\hline & $\begin{array}{l}\text { Dysphoria in chest, palms, and } \\
\text { soles }\end{array}$ & $\begin{array}{l}x x \\
(x x)\end{array}$ & $\begin{array}{l}x x \\
(x x)\end{array}$ &.$x x$ & $x x(x x)$ & $x x(x x)$ &.$x x$ \\
\hline & Dark purplish lips & $\begin{array}{l}x x \\
(x x)\end{array}$ & $\begin{array}{l}x x \\
(x x)\end{array}$ &.$x x$ & $x x(x x)$ & $x x(x x)$ &.$x x$ \\
\hline & Edema & $\begin{array}{l}x x \\
(x x)\end{array}$ & $\begin{array}{l}x x \\
(x x)\end{array}$ &.$x x$ & $x x(x x)$ & $x x(x x)$ &.$x x$ \\
\hline \multirow[t]{5}{*}{ Efficacy index } & Clinical control & $\begin{array}{l}x x \\
(x x \%)\end{array}$ & $\begin{array}{l}x x \\
(x x \%)\end{array}$ &.$x x$ & $x x(x x \%)$ & $x x(x x \%)$ &.$x x$ \\
\hline & Obvious therapeutic effect & $\begin{array}{l}x x \\
(x x \%)\end{array}$ & $\begin{array}{l}x x \\
(x x \%)\end{array}$ &.$x x$ & $x x(x x \%)$ & $x x(x x \%)$ &.$x x$ \\
\hline & With therapeutic effect & $\begin{array}{l}x x \\
(x x \%)\end{array}$ & $\begin{array}{l}x x \\
(x x \%)\end{array}$ & $x x$ & $x x(x x \%)$ & $x x(x x \%)$ &.$x x$ \\
\hline & Invalid & $\begin{array}{l}x x \\
(x x \%)\end{array}$ & $\begin{array}{l}x x \\
(x x \%)\end{array}$ &.$x x$ & $x x(x x \%)$ & $x x(x x \%)$ &.$x x$ \\
\hline & Overall clinical efficiency & $\begin{array}{l}x x \\
(x x \%)\end{array}$ & $\begin{array}{l}x x \\
(x x \%)\end{array}$ &.$x x$ & $x x(x x \%)$ & $x x(x x \%)$ &.$x x$ \\
\hline
\end{tabular}

Table 7. List of all adverse effects reported during the trial

\begin{tabular}{|lllll|}
\hline Description & SZF, number & Irbesartan, number & Severity & Related to trial \\
\hline$x x$ & $x x$ & $x x$ & $x x$ & $x x$ \\
$x x$ & $x x$ & $x x$ & $x x$ & $x x$ \\
$x x$ & $x x$ & $x x$ & $x x$ & $x x$ \\
\hline
\end{tabular}

Table 8. Safety assessment 


\begin{tabular}{|c|c|c|c|c|c|c|c|c|c|c|}
\hline & & \multicolumn{3}{|l|}{ ow } & \multicolumn{3}{|l|}{$12 W$} & \multicolumn{3}{|l|}{$24 W$} \\
\hline & & SZF & Irbesartan & $\begin{array}{l}p \\
\text { value }\end{array}$ & SZF & Irbesartan & $\begin{array}{l}p \\
\text { value }\end{array}$ & SZF & Irbesartan & $\begin{array}{l}p \\
\text { value }\end{array}$ \\
\hline & & $(n=x x)$ & $(n=x x)$ & & $(n=x x)$ & $(n=x x)$ & & $(n=x x)$ & $(n=x x)$ & \\
\hline \multirow[t]{4}{*}{ Haematology } & $\begin{array}{l}\text { Red blood } \\
\text { cell }\end{array}$ & $\begin{array}{l}x x \\
(x x)\end{array}$ & $x x(x x)$ &.$x x$ & $\begin{array}{l}x x \\
(x x)\end{array}$ & $x x(x x)$ &.$x x$ & $\begin{array}{l}x x \\
(x x)\end{array}$ & $x x(x x)$ &.$x x$ \\
\hline & $\begin{array}{l}\text { White blood } \\
\text { cell }\end{array}$ & $\begin{array}{l}x x \\
(x x)\end{array}$ & $x x(x x)$ &.$x x$ & $\begin{array}{l}x x \\
(x x)\end{array}$ & $x x(x x)$ &.$x x$ & $\begin{array}{l}x x \\
(x x)\end{array}$ & $x x(x x)$ &.$x x$ \\
\hline & Hemoglobin & $\begin{array}{l}x x \\
(x x)\end{array}$ & $x x(x x)$ &.$x x$ & $\begin{array}{l}x x \\
(x x)\end{array}$ & $x x(x x)$ &.$x x$ & $\begin{array}{l}x x \\
(x x)\end{array}$ & $x x(x x)$ &.$x x$ \\
\hline & Platelet & $\begin{array}{l}x x \\
(x x)\end{array}$ & $x x(x x)$ &.$x x$ & $\begin{array}{l}x x \\
(x x)\end{array}$ & $x x(x x)$ &.$x x$ & $\begin{array}{l}x x \\
(x x)\end{array}$ & $x x(x x)$ &.$x x$ \\
\hline \multirow[t]{5}{*}{ Urine test } & Glucose & $\begin{array}{l}x x \\
(x x)\end{array}$ & $x x(x x)$ &.$x x$ & $\begin{array}{l}x x \\
(x x)\end{array}$ & $x x(x x)$ &.$x x$ & $\begin{array}{l}x x \\
(x x)\end{array}$ & $x x(x x)$ &.$x x$ \\
\hline & Ketone & $\begin{array}{l}x x \\
(x x)\end{array}$ & $x x(x x)$ &.$x x$ & $\begin{array}{l}x x \\
(x x)\end{array}$ & $x x(x x)$ &.$x x$ & $\begin{array}{l}x x \\
(x x)\end{array}$ & $x x(x x)$ &.$x x$ \\
\hline & Occult blood & $\begin{array}{l}x x \\
(x x)\end{array}$ & $x x(x x)$ &.$x x$ & $\begin{array}{l}x x \\
(x x)\end{array}$ & $x x(x x)$ &.$x x$ & $\begin{array}{l}x x \\
(x x)\end{array}$ & $x x(x x)$ &.$x x$ \\
\hline & Erythrocytes & $\begin{array}{l}x x \\
(x x)\end{array}$ & $x x(x x)$ &.$x x$ & $\begin{array}{l}x x \\
(x x)\end{array}$ & $x x(x x)$ &.$x x$ & $\begin{array}{l}x x \\
(x x)\end{array}$ & $x x(x x)$ &.$x x$ \\
\hline & Leukocytes & $\begin{array}{l}x x \\
(x x)\end{array}$ & $x x(x x)$ &.$x x$ & $\begin{array}{l}x x \\
(x x)\end{array}$ & $x x(x x)$ &.$x x$ & $\begin{array}{l}x x \\
(x x)\end{array}$ & $x x(x x)$ &.$x x$ \\
\hline \multirow[t]{2}{*}{ Stool routine } & $\begin{array}{l}\text { Faecal } \\
\text { leukocytes }\end{array}$ & $\begin{array}{l}x x \\
(x x)\end{array}$ & $x x(x x)$ &.$x x$ & $\begin{array}{l}x x \\
(x x)\end{array}$ & $x x(x x)$ & $x x$ & $\begin{array}{l}x x \\
(x x)\end{array}$ & $x x(x x)$ &.$x x$ \\
\hline & $\begin{array}{l}\text { Feces occult } \\
\text { blood }\end{array}$ & $\begin{array}{l}x x \\
(x x)\end{array}$ & $x x(x x)$ &.$x x$ & $\begin{array}{l}x x \\
(x x)\end{array}$ & $x x(x x)$ &.$x x$ & $\begin{array}{l}x x \\
(x x)\end{array}$ & $x x(x x)$ &.$x x$ \\
\hline \multirow[t]{10}{*}{$\begin{array}{l}\text { Blood } \\
\text { chemistry }\end{array}$} & ALT & $\begin{array}{l}x x \\
(x x)\end{array}$ & $x x(x x)$ &.$x x$ & $\begin{array}{l}x x \\
(x x)\end{array}$ & $x x(x x)$ &.$x x$ & $\begin{array}{l}x x \\
(x x)\end{array}$ & $x x(x x)$ &.$x x$ \\
\hline & AST & $\begin{array}{l}x x \\
(x x)\end{array}$ & $x x(x x)$ &.$x x$ & $\begin{array}{l}x x \\
(x x)\end{array}$ & $x x(x x)$ &.$x x$ & $\begin{array}{l}x x \\
(x x)\end{array}$ & $x x(x x)$ &.$x x$ \\
\hline & ALP & $\begin{array}{l}x x \\
(x x)\end{array}$ & $x x(x x)$ &.$x x$ & $\begin{array}{l}x x \\
(x x)\end{array}$ & $x x(x x)$ &.$x x$ & $\begin{array}{l}x x \\
(x x)\end{array}$ & $x x(x x)$ &.$x x$ \\
\hline & GGT & $\begin{array}{l}x x \\
(x x)\end{array}$ & $x x(x x)$ & $x x$ & $\begin{array}{l}x x \\
(x x)\end{array}$ & $x x(x x)$ &.$x x$ & $\begin{array}{l}x x \\
(x x)\end{array}$ & $x x(x x)$ &.$x x$ \\
\hline & TBIL & $\begin{array}{l}x x \\
(x x)\end{array}$ & $x x(x x)$ &.$x x$ & $\begin{array}{l}x x \\
(x x)\end{array}$ & $x x(x x)$ &.$x x$ & $\begin{array}{l}x x \\
(x x)\end{array}$ & $x x(x x)$ &.$x x$ \\
\hline & UA & $\begin{array}{l}x x \\
(x x)\end{array}$ & $x x(x x)$ &.$x x$ & $\begin{array}{l}x x \\
(x x)\end{array}$ & $x x(x x)$ &.$x x$ & $\begin{array}{l}x x \\
(x x)\end{array}$ & $x x(x x)$ &.$x x$ \\
\hline & $\begin{array}{l}\beta 2- \\
\text { microglobulin }\end{array}$ & $\begin{array}{l}x x \\
(x x)\end{array}$ & $x x(x x)$ &.$x x$ & $\begin{array}{l}x x \\
(x x)\end{array}$ & $x x(x x)$ &.$x x$ & $\begin{array}{l}x x \\
(x x)\end{array}$ & $x x(x x)$ &.$x x$ \\
\hline & $\begin{array}{l}\text { Serum } \\
\text { albumin }\end{array}$ & $\begin{array}{l}x x \\
(x x)\end{array}$ & $x x(x x)$ &.$x x$ & $\begin{array}{l}x x \\
(x x)\end{array}$ & $x x(x x)$ &.$x x$ & $\begin{array}{l}x x \\
(x x)\end{array}$ & $x x(x x)$ &.$x x$ \\
\hline & Potassium & $\begin{array}{l}x x \\
(x x)\end{array}$ & $x x(x x)$ &.$x x$ & $\begin{array}{l}x x \\
(x x)\end{array}$ & $x x(x x)$ &.$x x$ & $\begin{array}{l}x x \\
(x x)\end{array}$ & $x x(x x)$ &.$x x$ \\
\hline & $\begin{array}{l}\text { Blood urea } \\
\text { nitrogen }\end{array}$ & $\begin{array}{l}x x \\
(x x)\end{array}$ & $x x(x x)$ &.$x x$ & $\begin{array}{l}x x \\
(x x)\end{array}$ & $x x(x x)$ &.$x x$ & $\begin{array}{l}x x \\
(x x)\end{array}$ & $x x(x x)$ &.$x x$ \\
\hline \multicolumn{2}{|c|}{ Electrocardiogram } & $\begin{array}{l}x x \\
(x x \%)\end{array}$ & $x x(x x \%)$ &.$x x$ & $\begin{array}{l}x x \\
(x x \%)\end{array}$ & $x x(x x \%)$ &.$x x$ & $\begin{array}{l}x x \\
(x x \%)\end{array}$ & $x x(x x \%)$ &.$x x$ \\
\hline
\end{tabular}




\begin{tabular}{|c|c|c|c|c|c|c|c|c|c|c|}
\hline $\begin{array}{l}\text { Adverse } \\
\text { events }\end{array}$ & Mild & $\begin{array}{l}x x \\
(x x \%)\end{array}$ & $x x(x x \%)$ &.$x x$ & $\begin{array}{l}x x \\
(x x \%)\end{array}$ & $x x(x x \%)$ &.$x x$ & $\begin{array}{l}x x \\
(x x \%)\end{array}$ & $x x(x x \%)$ &.$x x$ \\
\hline & Moderate & $\begin{array}{l}x x \\
(x x \%)\end{array}$ & $x x(x x \%)$ &.$x x$ & $\begin{array}{l}x x \\
(x x \%)\end{array}$ & $x x(x x \%)$ &.$x x$ & $\begin{array}{l}x x \\
(x x \%)\end{array}$ & $x x(x x \%)$ &.$x x$ \\
\hline & Severe & $\begin{array}{l}x x \\
(x x \%)\end{array}$ & $x x(x x \%)$ &.$x x$ & $\begin{array}{l}x x \\
(x x \%)\end{array}$ & $x x(x x \%)$ &.$x x$ & $\begin{array}{l}x x \\
(x x \%)\end{array}$ & $x x(x x \%)$ &.$x x$ \\
\hline & Overall AEs & $\begin{array}{l}x x \\
(x x \%)\end{array}$ & $x x(x x \%)$ &.$x x$ & $\begin{array}{l}x x \\
(x x \%)\end{array}$ & $x x(x x \%)$ &.$x x$ & $\begin{array}{l}x x \\
(x x \%)\end{array}$ & $x x(x x \%)$ &.$x x$ \\
\hline
\end{tabular}

\section{Study Period}

\begin{tabular}{|c|c|c|c|c|c|c|c|}
\hline \multicolumn{8}{|l|}{ study period } \\
\hline & enrollment & allocation & interver & & & & \\
\hline Time point(weeks) & -2 & 0 & 48 & 12 & 16 & 20 & 24 \\
\hline Eligibility screen & $\sqrt{ }$ & & & & & & \\
\hline Written informed consent & & $\sqrt{ }$ & & & & & \\
\hline Physical examination & $\sqrt{ }$ & & & & & & \\
\hline Medical history & $\sqrt{ }$ & & & & & & \\
\hline $24 \mathrm{~h}$ urinary protein & $\sqrt{ }$ & $\sqrt{ }$ & $\sqrt{ } \quad \sqrt{ }$ & $\sqrt{ }$ & $\sqrt{ }$ & $\sqrt{ }$ & $\sqrt{ }$ \\
\hline UAER』ACR & & $\sqrt{ }$ & $\sqrt{ } \quad \sqrt{ }$ & $\sqrt{ }$ & $\sqrt{ }$ & $\sqrt{ }$ & $\sqrt{ }$ \\
\hline FBG, $2 \mathrm{hPG}$ & $\sqrt{ }$ & $\sqrt{ }$ & & $\sqrt{ }$ & & & $\sqrt{ }$ \\
\hline $\mathrm{HbA1c}$ & $\sqrt{ }$ & $\sqrt{ }$ & $\sqrt{ } \quad \sqrt{ }$ & $\sqrt{ }$ & $\sqrt{ }$ & $\sqrt{ }$ & $\sqrt{ }$ \\
\hline $\mathrm{BP}$ & $\sqrt{ }$ & $\sqrt{ }$ & $\sqrt{ } \quad \sqrt{ }$ & $\sqrt{ }$ & $\sqrt{ }$ & $\sqrt{ }$ & $\sqrt{ }$ \\
\hline TC, TG, LDL-C, HDL-C & & $\sqrt{ }$ & & $\sqrt{ }$ & & & $\sqrt{ }$ \\
\hline Routine blood and CRP,urine, and stool & & $\sqrt{ }$ & & $\sqrt{ }$ & & & $\sqrt{ }$ \\
\hline ALT,AST, ALP GGL, TBIL & & $\sqrt{ }$ & & $\sqrt{ }$ & & & $\sqrt{ }$ \\
\hline BUN, Serum Cr, $\beta 2-M G, U A$ & & $\sqrt{ }$ & $\sqrt{ } \quad \sqrt{ }$ & $\sqrt{ }$ & $\sqrt{ }$ & $\sqrt{ }$ & $\sqrt{ }$ \\
\hline Electrocardiogram & & $\sqrt{ }$ & & $\sqrt{ }$ & & & $\sqrt{ }$ \\
\hline Adverse events & & $\sqrt{ }$ & $\sqrt{ } \quad \sqrt{ }$ & $\sqrt{ }$ & $\sqrt{ }$ & $\sqrt{ }$ & $\sqrt{ }$ \\
\hline ADDQoL & & $\sqrt{ }$ & & $\sqrt{ }$ & & & $\sqrt{ }$ \\
\hline
\end{tabular}

Figures 


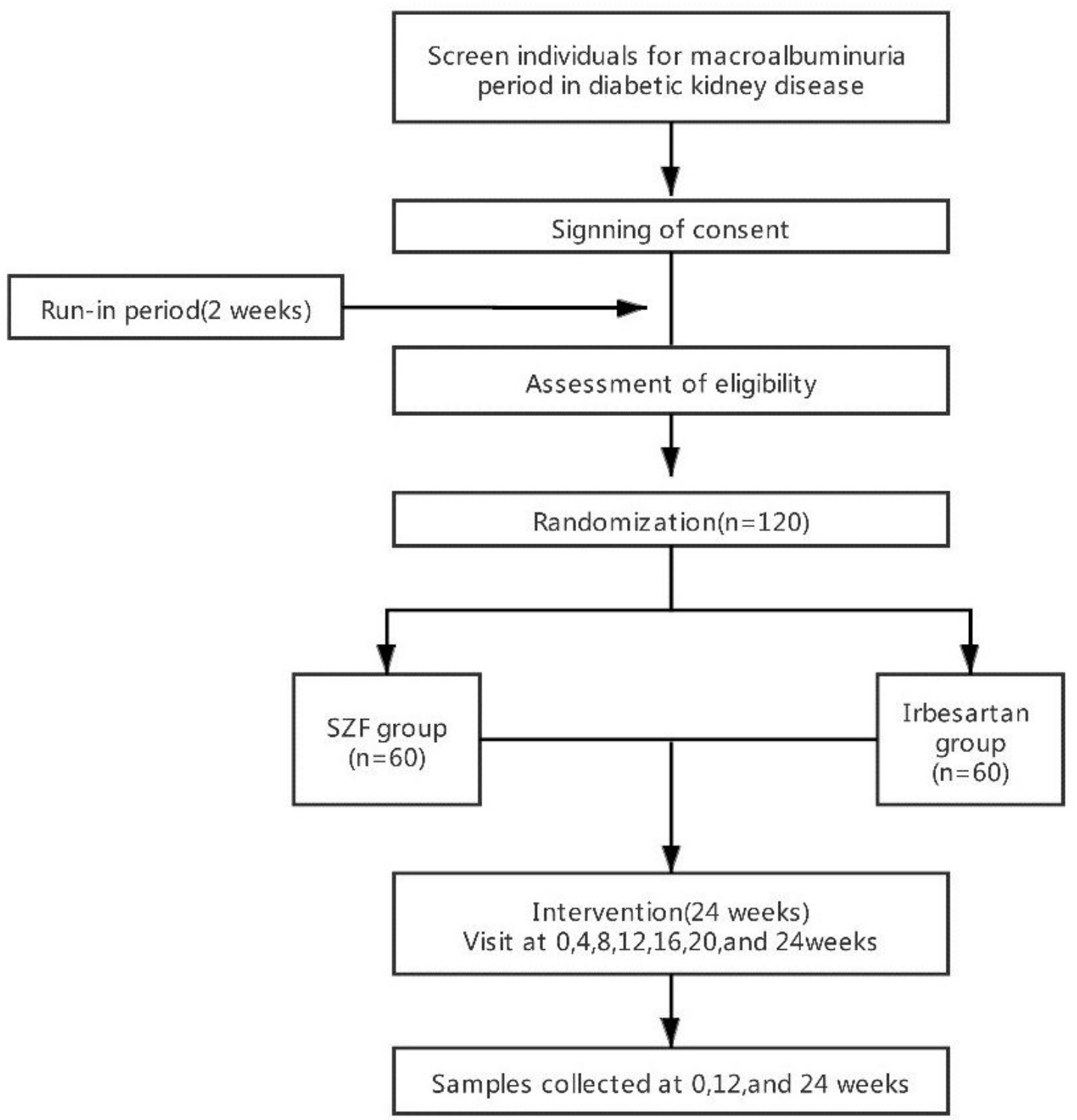

Figure 1

Flow diagram of the study

\section{Supplementary Files}

This is a list of supplementary files associated with this preprint. Click to download.

- AdditionalFile1.docx 